\title{
Article \\ MiR-10a in Pancreatic Juice as a Biomarker for Invasive Intraductal Papillary Mucinous Neoplasm by miRNA Sequencing
}

\author{
Natsuhiko Kuratomi ${ }^{1}$, Shinichi Takano ${ }^{1, *(D)}$, Mitsuharu Fukasawa ${ }^{1}$, Shinya Maekawa ${ }^{1}$ (D), Makoto Kadokura ${ }^{1}$, \\ Hiroko Shindo ${ }^{1}$, Ei Takahashi ${ }^{1}$, Sumio Hirose ${ }^{1}$, Yoshimitsu Fukasawa ${ }^{1}$, Satoshi Kawakami ${ }^{1}$, \\ Hiroshi Hayakawa ${ }^{1}$, Hitomi Takada ${ }^{1}$, Natsuko Nakakuki ${ }^{1}$, Ryoh Kato ${ }^{1}$, Tatsuya Yamaguchi ${ }^{1}$, \\ Yasuhiro Nakayama ${ }^{1}$, Hiromichi Kawaida ${ }^{2}$, Hiroshi Kono ${ }^{2}$, Taisuke Inoue ${ }^{1}$, Tetsuo Kondo ${ }^{3}$, Daisuke Ichikawa ${ }^{2}$ \\ and Nobuyuki Enomoto ${ }^{1}$
}

check for updates

Citation: Kuratomi, N.; Takano, S.;

Fukasawa, M.; Maekawa, S.;

Kadokura, M.; Shindo, H.; Takahashi,

E.; Hirose, S.; Fukasawa, Y.;

Kawakami, S.; et al. MiR-10a in

Pancreatic Juice as a Biomarker for

Invasive Intraductal Papillary

Mucinous Neoplasm by miRNA

Sequencing. Int. J. Mol. Sci. 2021, 22,

3221. https://doi.org/10.3390/

ijms 22063221

Academic Editors: Paola Ghiorzo and Tianhua Niu

Received: 5 February 2021

Accepted: 19 March 2021

Published: 22 March 2021

Publisher's Note: MDPI stays neutral with regard to jurisdictional claims in published maps and institutional affiliations.

Copyright: (c) 2021 by the authors Licensee MDPI, Basel, Switzerland. This article is an open access article distributed under the terms and conditions of the Creative Commons Attribution (CC BY) license (https:// creativecommons.org/licenses/by/ $4.0 /)$
1 First Department of Internal Medicine, Faculty of Medicine, University of Yamanashi, Chuo, Yamanashi 409-3898, Japan; nkuratomi@yamanashi.ac.jp (N.K.); fmitsu@yamanashi.ac.jp (M.F.); maekawa@yamanashi.ac.jp (S.M.); makotok@yamanashi.ac.jp (M.K.); shindoh@yamanashi.ac.jp (H.S.); etakahashi@yamanashi.ac.jp (E.T.); sh99073@yahoo.co.jp (S.H.); ii258pp2@yahoo.co.jp (Y.F.); skawakami@yamanashi.ac.jp (S.K.); minaminoro73@gmail.com (H.H.); thitomi@yamanashi.ac.jp (H.T.); natsukon@yamanashi.ac.jp (N.N.); katohr@yamanashi.ac.jp (R.K.); ytatsuya@yamanashi.ac.jp (T.Y.); ynakayama@yamanashi.ac.jp (Y.N.); tinoue@yamanashi.ac.jp (T.I.); enomoto@yamanashi.ac.jp (N.E.)

2 First Department of Surgery, Faculty of Medicine, University of Yamanashi, Chuo, Yamanashi 409-3898, Japan; kawaidah@yamanashi.ac.jp (H.K.); hkouno@yamanashi.ac.jp (H.K.); dichikawa@yamanashi.ac.jp (D.I.)

3 Department of Pathology, Faculty of Medicine, University of Yamanashi, Chuo, Yamanashi 409-3898, Japan; ktetsuo@yamanashi.ac.jp

* Correspondence: stakano@yamanashi.ac.jp; Tel.: +81-55-273-9584

Abstract: New biomarkers are needed to further stratify the risk of malignancy in intraductal papillary mucinous neoplasm (IPMN). Although microRNAs (miRNAs) are expected to be stable biomarkers, they can vary owing to a lack of definite internal controls. To identify universal biomarkers for invasive IPMN, we performed miRNA sequencing using tumor-normal paired samples. A total of 19 resected tissues and 13 pancreatic juice samples from 32 IPMN patients were analyzed for miRNA expression by next-generation sequencing with a two-step normalization of miRNA sequence data. The miRNAs involved in IPMN associated with invasive carcinoma were identified from this tissue analysis and further verified with the pancreatic juice samples. From the tumor-normal paired tissue analysis of the expression levels of 2792 miRNAs, 20 upregulated and 17 downregulated miRNAs were identified. In IPMN associated with invasive carcinoma (INV), miR-10a-5p and miR-221-3p were upregulated and miR-148a-3p was downregulated when compared with noninvasive IPMN. When these findings were further validated with pancreatic juice samples, miR-10a-5p was found to be elevated in INV ( $p=0.002)$. Therefore, three differentially expressed miRNAs were identified in tissues with INV, and the expression of miR-10a-5p was also elevated in pancreatic juice samples with INV. MiR-10a-5p is a promising additional biomarker for invasive IPMN.

Keywords: intraductal papillary mucinous neoplasm; miRNA; pancreatic juice; formalin-fixed paraffin-embedded samples; next-generation sequencing

\section{Introduction}

Intraductal papillary mucinous neoplasm (IPMN) is a noninvasive mucin-producing epithelial neoplasm, which is grossly visible, predominantly papillary, and rarely flat and which grows in the main or branch pancreatic ducts [1,2]. The prevalence of incidental findings of IPMN or pancreatic cyst increased to $4.3 \%$ with improved diagnostic imaging technologies [3,4]. IPMN, like pancreatic intraepithelial neoplasia (PanIN), is a lesion, which is a precursor of pancreatic ductal carcinoma, and shows a wide-ranging histological 
spectrum, from low-grade dysplasia (LGD) and high-grade dysplasia (HGD) to IPMN with an associated invasive carcinoma (INV) [1,5]. IPMN with INV exhibits a poor survival rate after resection when the tumor is accompanied by lymph node metastases or histological findings of tubular adenocarcinoma [6,7]. The five-year survival rate of INV after resection (30\%) is much poorer than that of LGD and HGD (73\% and 70\%, respectively) [8]. Therefore, diagnosing INV without delay is extremely important.

To provide an optimal management algorithm for IPMN, the International Consensus Guidelines (ICG) for the management of IPMN and mucinous cystic neoplasms of the pancreas were established and revised in 2006 and $2012[9,10]$ and most recently in 2017 [11]. The ICG stratify risks for malignant IPMN by mainly using the mural nodule size, pancreatic duct diameter and cyst size, which can also be assessed with regular imaging tests, such as computed tomography, magnetic resonance imaging and endoscopic ultrasonography. The sensitivity of diagnosing malignant IPMN, including HGD and INV, has improved with the revisions of the ICG to more than $90 \%$; however, the specificity remains less than $30 \%$, which can lead to overtreatment in patients with IPMN [12]. Further, $9 \%$ of patients with malignant IPMN would be treated as low risk, and their diagnoses would be missed [12-14]. Conversely, pancreaticoduodenectomy and total pancreatectomy are relatively invasive treatments with treatment-related mortalities of $0-4.9 \%$ and $0-8 \%$, respectively, and they require careful indication [15-17]. Additional malignant predictors, including new biomarkers, are required to select an optimal treatment for IPMN.

Recent developments in biological analysis made amplifying target genes and performing a comprehensive gene analysis by DNA and RNA sequencing possible even from unstable and small clinical samples, including pancreatic tissues and pancreatic juice samples [18-20]. Compared with the use of other biological molecules such as proteins, DNAs and other RNAs as biomarkers, microRNA (miRNA) demonstrates the advantage of being very stable $[21,22]$. MiRNAs are small, noncoding RNAs consisting typically of 18-23 nucleotides, which can base-pair with complementary sequences in mRNA to silence RNA and post-transcriptionally regulate gene expression [23], such as during tumorigenesis and metastasis [24]. The molecular functions [25,26] and usefulness of these molecules as potential biomarkers [27-30] in pancreatic cancer have been reported, which suggests that new miRNA biomarkers for malignant IPMN could be found. However, one disadvantage of miRNAs as biomarkers is their lack of a definite internal control, which leads to inconsistent results across different studies.

In this study, in order to identify miRNA biomarkers for malignant IPMN, we comprehensively analyzed the miRNA expression using next-generation sequencing (NGS) in tumor tissues paired with normal tissues and validated the usefulness of these biomarkers using pancreatic juice samples.

\section{Results}

\subsection{Quantification of miRNAs Extracted from Clinical Samples}

MiRNA sequencing was performed, and an average of 36,485 and 34,168 miRNA reads from the sequence reads of formalin-fixed paraffin-embedded (FFPE) and pancreatic juice, respectively, were mapped to the reference genome (hg19) (Table S1). Of 2792 total miRNAs, we eliminated immature miRNAs and miRNAs with extremely low expression levels (no more than an average of three reads), leaving 194 and 162 miRNAs from tissue and pancreatic juice samples, respectively, for subsequent analysis (Table S1).

\subsection{Differential miRNA Expression in IPMN by NGS Analysis}

After normalization of miRNA sequence reads by calculating the percentage of reads in the total sample for each miRNA, we further normalized the results for 194 miRNAs by calculating the ratio of the miRNA expression in the tumor tissue to the paired normal acinar tissue in 19 tumor-normal paired tissue samples (two-step normalization). Next, we performed a volcano plot analysis with paired t-tests and fold changes of miRNA expressions, and we revealed 37 miRNAs that were differentially expressed between tumor and 
normal acinus tissues (20 upregulated and 17 downregulated in tumor tissue) (Figure 1a). Next, we further revealed 11 miRNAs that were differentially expressed in tumor tissues compared with normal acinar tissues in LGD (nine upregulated, two downregulated), 36 in HGD (19 upregulated, 17 downregulated) and 41 in INV (15 upregulated, 26 downregulated) (Figure 1b-d), with a tendency for an increase in the number of differentially expressed miRNAs with an increased histological malignancy. Overall, we identified 69 unique differentially expressed miRNAs.

a

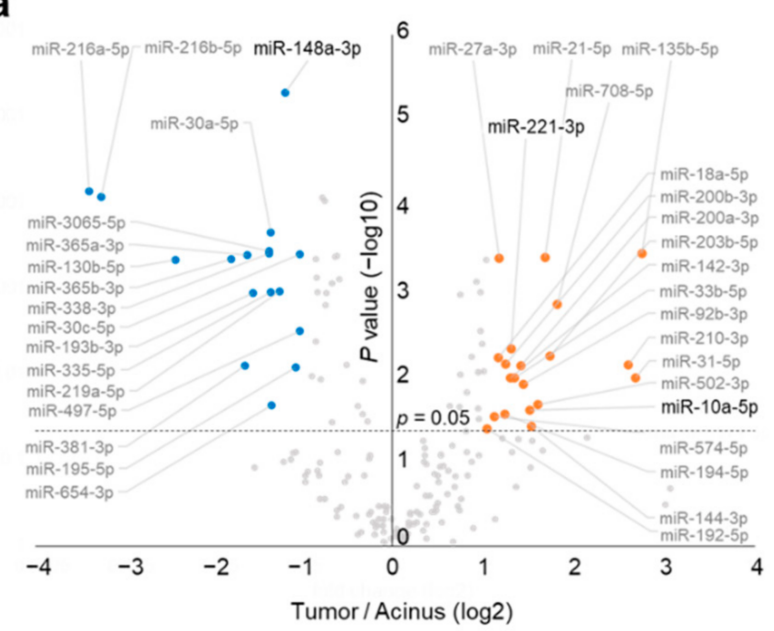

C

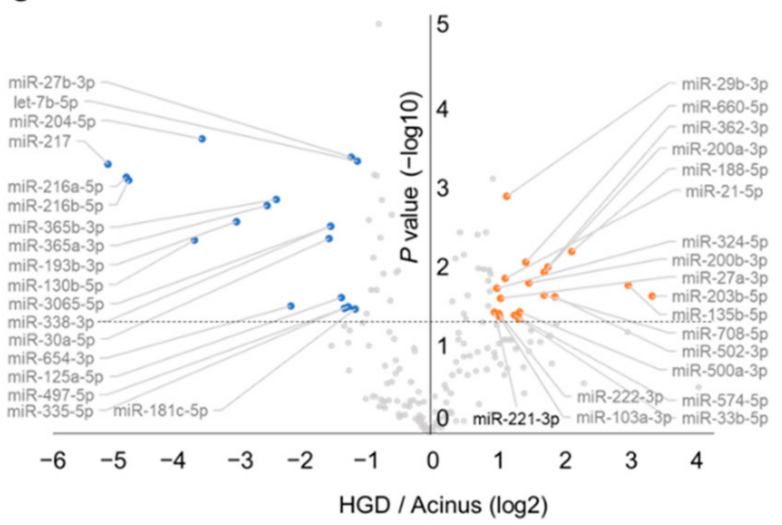

b

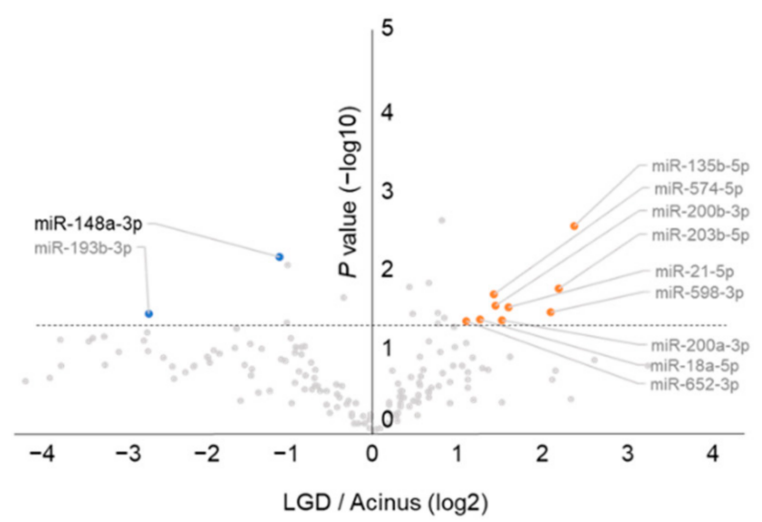

d

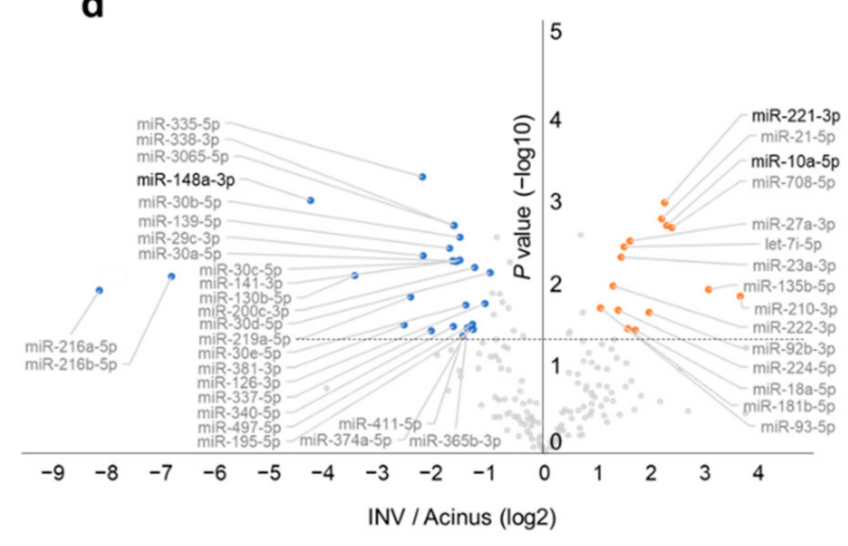

Figure 1. Volcano plots of next-generation sequencing data show differential miRNA expressions in intraductal papillary mucinous neoplasm (IPMN) between tumor tissue and its paired normal acinus tissue. (a) All IPMN cases $(n=19)$. (b) Cases with low-grade dysplasia (LGD) $(n=4)$. (c) Cases with high-grade dysplasia (HGD) $(n=9)$. (d) Cases with invasive carcinoma (INV) $(n=6)$. Orange dots show significantly upregulated miRNAs (fold-change $>2, p<0.05$ ), and blue dots show significantly downregulated miRNAs (fold-change $<0.5, p<0.05$ ).

\subsection{MiR-10a and miR-221 were Upregulated in Invasive IPMN}

Next, we compared the 69 differentially expressed miRNAs between the three histological grades and showed the difference of miRNA expressions among histological grades as a heatmap with $p$-values (Figure 2). Statistical analyses of the tumor: the normal ratios of the miRNA expression levels among the three grades revealed 15 differentially expressed miRNAs (miR-210-3p, miR-221-3p, let-7i-5p, miR-10a-5p, miR-598-3p, miR-200a-3p, miR200b-3p, miR-200c-3p, miR-660-5p, miR-362-3p, miR-29c-3p, miR-340-5p, miR-148a-3p, let-7b-5p and miR-193-3p), as shown in Figure 2. Further, we identified that miR-10a-5p (Figure 3a,d) and miR-221-3p (Figure 3b,e) were upregulated in invasive IPMN (INV) when compared with noninvasive IPMN (LGD and HGD) and that miR-148a-3p (Figure 3c,f) was downregulated in noninvasive IPMN by post hoc analyses, which compared each of two 
groups after a comparison of the three groups (Table 1). No miRNAs were differentially expressed between LGD and HGD.

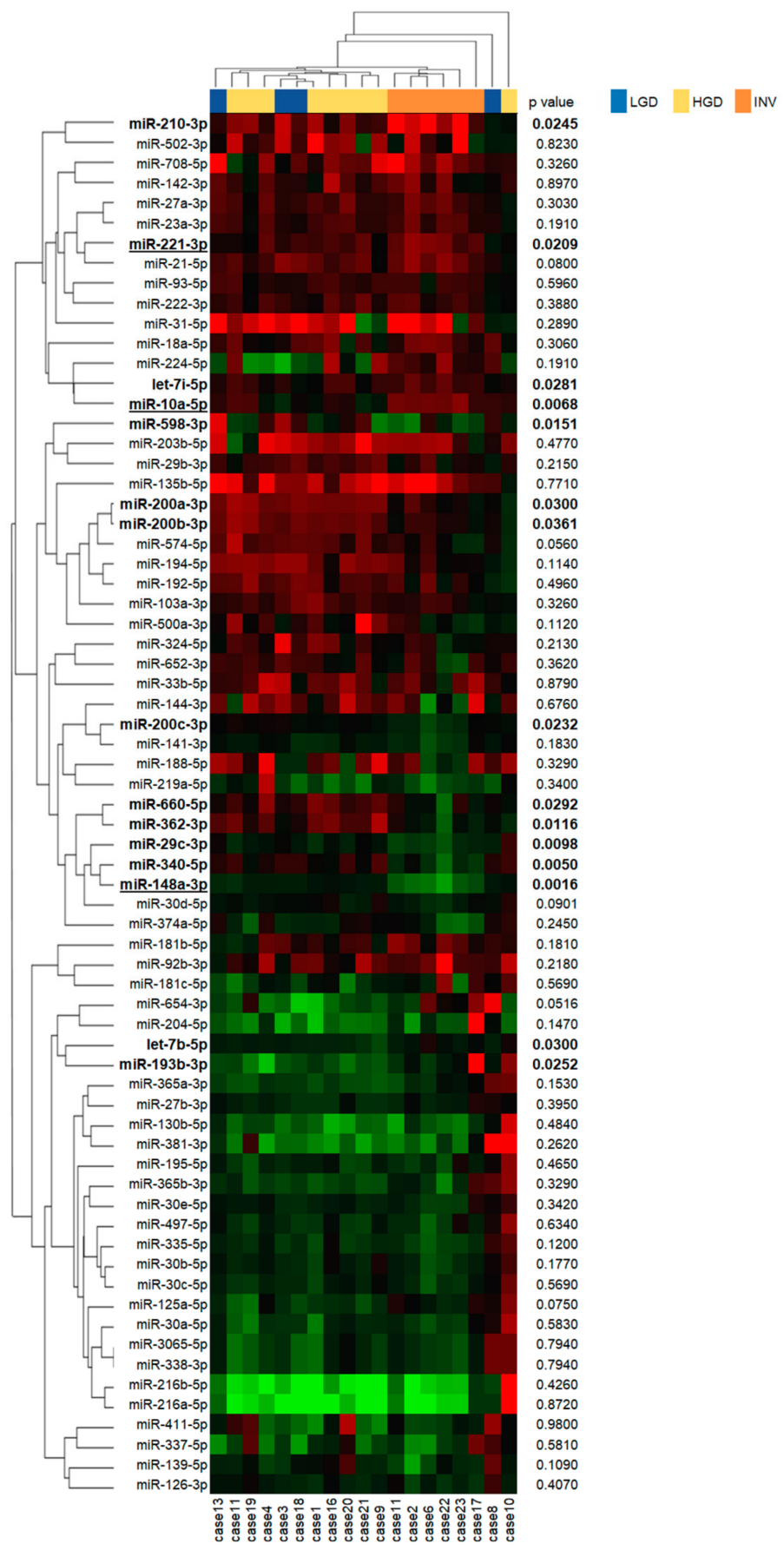

Figure 2. Heatmap of miRNA expressions stratified by pathological grades of intraductal papillary mucinous neoplasm (IPMN). The expressions of each miRNA in the IPMN tissues are shown as a heatmap. The expression levels of miRNAs are shown on a green-red scale in the center panel where the green and red should be interpreted as a low and high expression, respectively. The right panel shows the $p$-values by a Kruskal-Wallis test comparing three pathological grades (bold $p$ value $<0.05)$. The left and upper panels show miRNAs and patients that were arranged according to an unsupervised hierarchical clustering, which was performed using a one minus Pearson's correlation as the distance measure and an average linkage as the agglomerative method. LGD, low-grade dysplasia; HGD, high-grade dysplasia; INV, invasive carcinoma. MiRNAs with underlines show significant differential expressions between INV and non-INV (LGD and HGD) patients. 

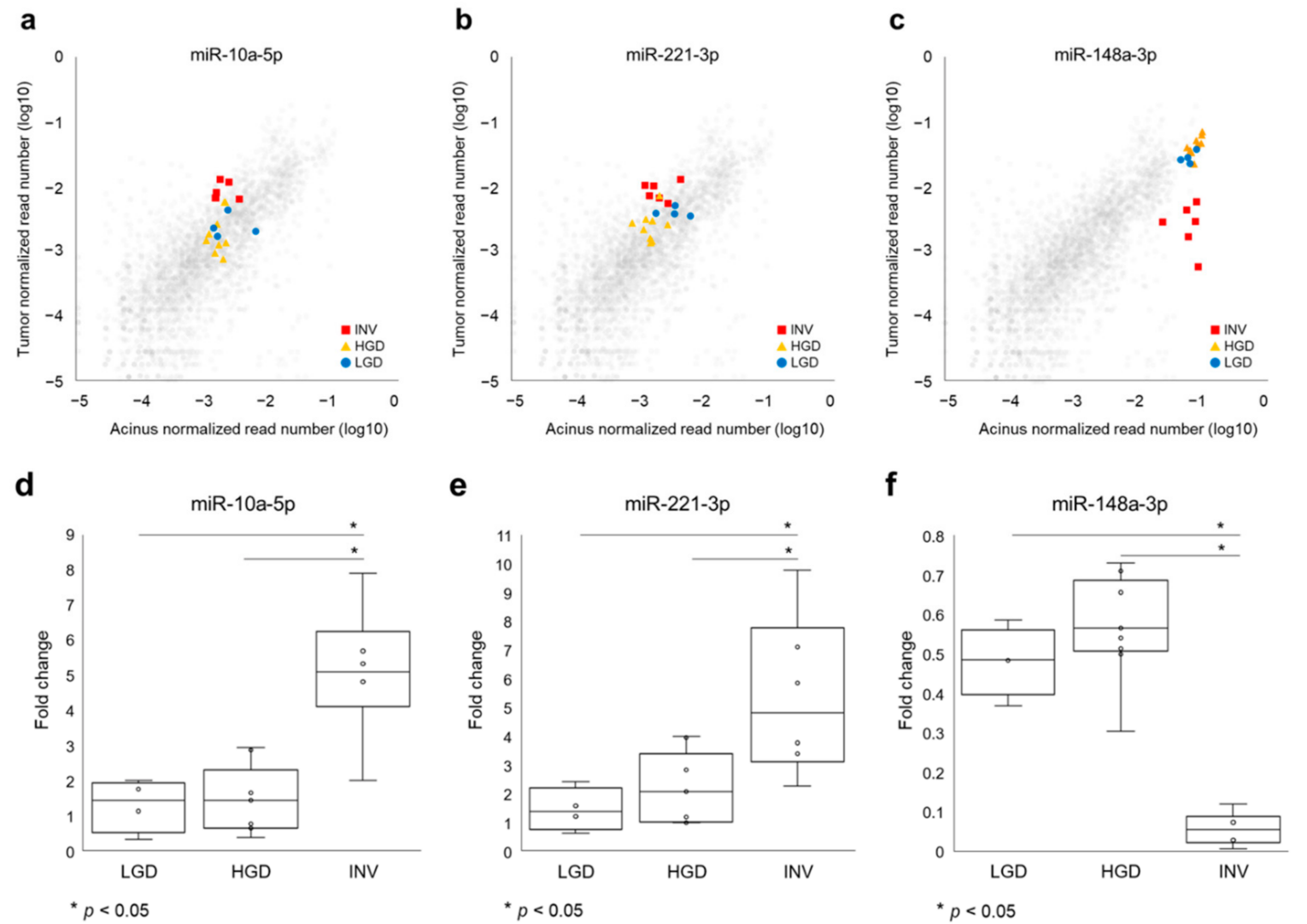

Figure 3. Candidate biomarker miRNAs in intraductal papillary mucinous neoplasm (IPMN) tissues to differentiate invasive IPMNs from noninvasive IPMNs. (a,d) MiR-10a-5p and (b,e) miR-221-3p were significantly upregulated in invasive carcinoma (INV) compared to low-grade dysplasia (LGD) and high-grade dysplasia (HGD). (c,f) MiR-148-3p was downregulated in INV compared to LGD and HGD. Gray dots indicate all other normalized read numbers of miRNA for each grade.

Table 1. A post hoc analysis of 15 microRNAs that exhibited significantly different expression patterns among three histological grades (Kruskal-Wallis test, $p<0.05$ ).

\begin{tabular}{cccc}
\hline & LGD vs. HGD & LGD vs. INV & HGD vs. INV \\
\cline { 2 - 4 } & $p$ Value & $p$ Value & $p$ Value \\
\hline miR-210-3p & 0.949 & 0.133 & $0.026^{*}$ \\
miR-221-3p & 0.811 & $0.049^{*}$ & $0.048^{*}$ \\
let-7i-5p & 0.271 & $0.028^{*}$ & 0.276 \\
miR-10a-5p & 1 & $0.049^{*}$ & $0.009^{*}$ \\
miR-598-3p & 0.078 & $0.028^{*}$ & 0.333 \\
miR-200a-3p & 0.624 & 0.203 & $0.036^{*}$ \\
miR-200b-3p & 0.889 & 0.203 & $0.036^{*}$ \\
miR-200c-3p & 0.889 & 0.083 & $0.036^{*}$ \\
miR-660-5p & 0.347 & 0.407 & $0.036^{*}$ \\
miR-362-3p & 0.206 & 0.133 & $0.026^{*}$ \\
miR-29c-3p & 0.811 & 0.083 & $0.026^{*}$ \\
miR-340-5p & 0.949 & 0.083 & $0.009^{*}$ \\
miR-148a-3p + & 0.271 & $0.028^{*}$ & $0.004^{*}$ \\
let-7b-5p & 0.526 & 0.133 & $0.048^{*}$ \\
miR-193b-3p & 0.624 & 0.133 & $0.036^{*}$
\end{tabular}

LGD, low-grade dysplasia; HGD, high-grade dysplasia; INV, invasive IPMN; ${ }^{*}, p<0.05 ;+$, Statistically significant in both LGD vs. INV and HGD vs. INV. 


\subsection{MiR-10a was Revealed to Be a Malignant Biomarker of IPMN in Pancreatic Juice}

We validated these identified miRNAs in preoperative pancreatic juice samples, which were taken from near the tumor and had the potential to contain biomarkers for malignant IPMNs. A volcano plot analysis of miRNAs in pancreatic juice samples revealed that five miRNAs (miR-10a-5p, miR-106b-5p, miR-197-3p, miR-664a-3p and let-7d-3p) were upregulated in IPMN associated with INV, compared with LGD and HGD. Of the miRNAs that were elevated in tissue samples, miR-10a-5p was upregulated in IPMN associated with INV when compared with HGD or LGD ( $p=0.002$, fold-change; 7.2 , Figure 4a), while $\mathrm{miR}-221-3 \mathrm{p}$ only tended to be elevated and was not statistically different, likely due to the small sample size of samples from patients with IPMN associated with INV (Figure $4 b$ ). Overall, miR-10a-5p seemed to be a useful and biologically important biomarker.

a

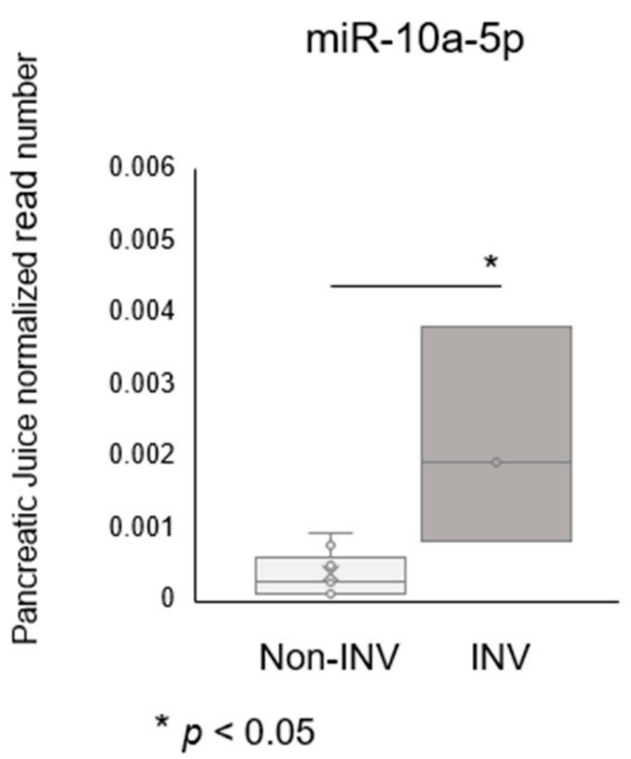

b

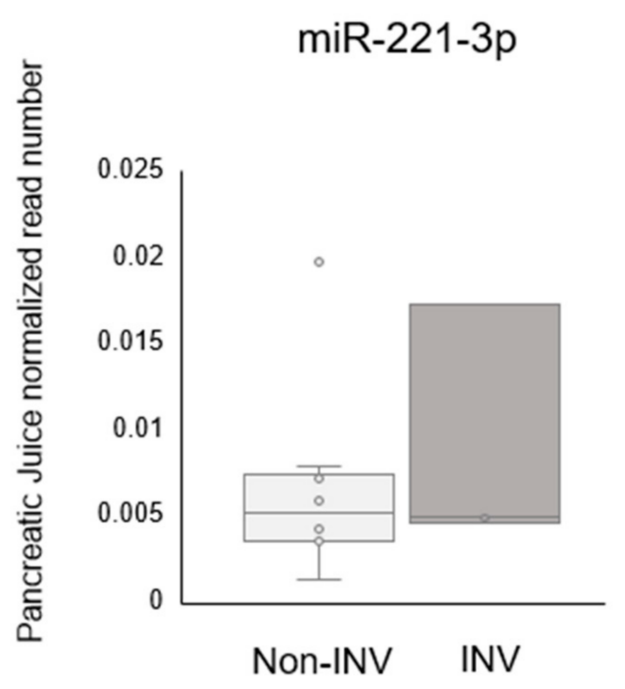

Figure 4. The miRNA expression of candidate biomarkers in intraductal papillary mucinous neoplasm (IPMN) pancreatic juice samples. (a) MiR-10a-5p was significantly upregulated in invasive IPMN compared to noninvasive IPMN, and (b) miR-221-5p showed a similar tendency. INV, invasive carcinoma.

\section{Discussion}

In this study, we showed that miR-10a and miR-221 were upregulated and that miR148a was downregulated in IPMN associated with invasive carcinoma by NGS using tumornormal paired resected tissues. In addition, we validated this finding by showing that miR-10a was upregulated in pancreatic juice samples from IPMN associated with invasive carcinomas, and it is a promising biomarker candidate for diagnosing malignant IPMN.

We identified miRNA biomarkers that were not previously reported in IPMN by performing an NGS analysis using tumor-normal paired tissues. A few reports [31-34] have detected differentially expressed miRNAs in IPMN, none of which reported elevated levels of miR-10a and miR-221 in malignant IPMN. Previous analyses of candidate miRNAs in IPMN reported that miR-21 and miR-155 were upregulated in IPMN [31] and invasive IPMN [32]. Comprehensive miRNA analyses revealed other miRNA biomarkers for malignant IPMN, such as miR-100, miR-99a, miR-99b, miR-342-3p, miR-126, miR-130a, miR-217, miR-216a, miR-216b, miR-148a, miR-375, miR-130b, miR-146b, miR-150, miR-214, miR-503, miR-21, miR-708 and miR-155 using microarrays [33,35], and miR-216a, miR-217, miR-802, miR-204, miR-218-1 and miR-214 using NGS [36]. Most of the obtained miRNAs demonstrated interstudy variations. One possible reason for the variation of results among 
studies was that internal controls for miRNA were not established, and another is that the background miRNA expression levels in each individual are likely to exhibit a strong effect on results. To overcome these problems, we performed a two-step normalization of the miRNA sequence data by, first, calculating the count data percentage for each miRNA per sample and then calculating the ratio of expression in the tumor and the adjacent acinus normal tissue. We believe that this two-step normalization enabled the validation of two miRNA biomarkers, miR-10a and miR-221, which were derived from tissue and pancreatic juice samples.

The newly identified biomarkers in IPMN, miR-10a and miR-221 are already known to be biologically meaningful miRNAs in other neoplasms. MiR-10a is upregulated in various tumors, including pancreatic cancer [37], especially in malignant and metastatic tumors [38-40], and exhibits functions, such as tumor migration and invasion through PTEN [41,42] and HOXA1 [43]. This led us to presume that miR-10a was involved in the progression function of tumors as an oncomiR. MiR-221 also exhibits a progressive function in various tumors and interferes with tumor suppressor genes, such as PTEN, CDKN1B and BLCL2L11 in pancreatic cancer cell lines [44-46]. These two miRNAs are also promising biomarkers; miR-10a may predict the advanced stage and prognosis in acute myeloid leukemia [47] and cervical cancer [48], and the development of pancreatic cancer [49]. miR-221 is also a candidate biomarker for pancreatic cancer [50]. These reports support the significance of our findings on miR-10a and miR-221 as clinically and biologically important biomarkers.

Multiple clinical implications of our study's findings exist. First, new biomarkers for malignant IPMN should be validated in other cohorts and analyzed in subgroups depending on radiological morphologies, histological grades and epithelial types of IPMN, which could be added to the ICG in order to attain an improved risk stratification of IPMN. Second, our two-step normalization of the miRNA sequences should be validated in other studies. Although miRNAs are expected to be good biomarkers in various diseases because of their stable expression, the lack of definite internal controls remains a problem for their analysis. In this study, we provided a two-step normalization method to analyze miRNA sequence read data, which we believe will improve the universality of the obtained results. Third, due to their stable expression, even in poorly conditioned clinical samples that contain many enzymes, miRNAs are expected to be useful biomarkers in liquid biopsies. We showed that this was feasible in pancreatic juice samples, and we also expect miRNAs to be of clinical use in the future in samples such as plasma, duodenal juice and ascites.

This study has several limitations. First, the design was retrospective, and hence only a small number of cases were recruited from a single center. Therefore, we could not further analyze the associations between miRNA expressions, morphological classification and IPMN subtypes, although we recognize the importance of these analyses. Second, the miRNA analysis in pancreatic juice was performed only for miR-10a and miR-221 in order to avoid multiple statistical tests in a small sample.

In conclusion, this study provides new miRNA biomarkers for malignant IPMN via a miRNA sequence using tumor-normal paired tissues. After these biomarkers are validated by large cohorts with subgroups based on the radiological morphology, histological grades and epithelial type of IPMN, we hope that these biomarkers will provide additional benefits to the international consensus guideline for a better risk stratification of IPMN.

\section{Materials and Methods}

\subsection{Patients and Samples}

We retrospectively analyzed resected tissues and pancreatic juice samples of 40 patients with IPMN who received surgical resections and/or systemic chemotherapy at Yamanashi University Hospital between September 2009 and November 2019. The study flow chart is shown in Figure 5, and the clinical characteristics of the enrolled patients are shown in Table 2 and Table S2. Tissues were obtained from resected specimens in which tumor components and their adjacent normal acini were separated by laser capture 
microdissection (LCM) using an ArcturusXT Laser Capture Microdissection System (Life Technologies, Carlsbad, CA, USA) from 8- $\mu$ m thick sections of FFPE samples. Representative histological images of LGD, HGD and invasive IPMN (INV) are shown in Figure S1. Samples were excluded if a paired normal acinus sample was not available. The tumor tissues and their paired normal acini were subjected to comprehensive miRNA analysis by NGS $(n=19)$. According to the manufacturer's specifications, the total RNA including miRNA was extracted from LCM specimens with the AllPrep DNA/RNA FFPE Kit (QIAGEN, Hilden, Germany). Pancreatic juice samples were collected via preoperative endoscopic retrograde pancreatography from 18 patients who underwent surgical resection between 2009 and 2013. The patients whose tissues were analyzed were an independent cohort from those whose pancreatic juice samples were analyzed. MiRNAs were extracted using the miRNeasy Mini Kit (QIAGEN, Hilden, Germany) from $500 \mu \mathrm{L}$ of pancreatic juice samples. Of the 18 pancreatic juice samples, five were excluded from the analysis because of few sequence reads in the downstream NGS analysis (Figure 5). The quantity and quality of the extracted RNA was assessed by a NanoDrop (Thermo Fisher, Waltham, MA, USA) instrument with the Qubit platform (Thermo Fisher, Waltham, MA, USA), and the small RNA fraction including miRNA was assessed by an RNA 6000 Nano Kit on the Agilent 2100 Bioanalyzer on-chip electrophoresis (Agilent Technologies, Santa Clara, CA, USA). This study was approved by the Human Ethics Review Committee of Yamanashi University Hospital (Receipt numbers: 1326 and 1847), and written informed consent was obtained from all patients.
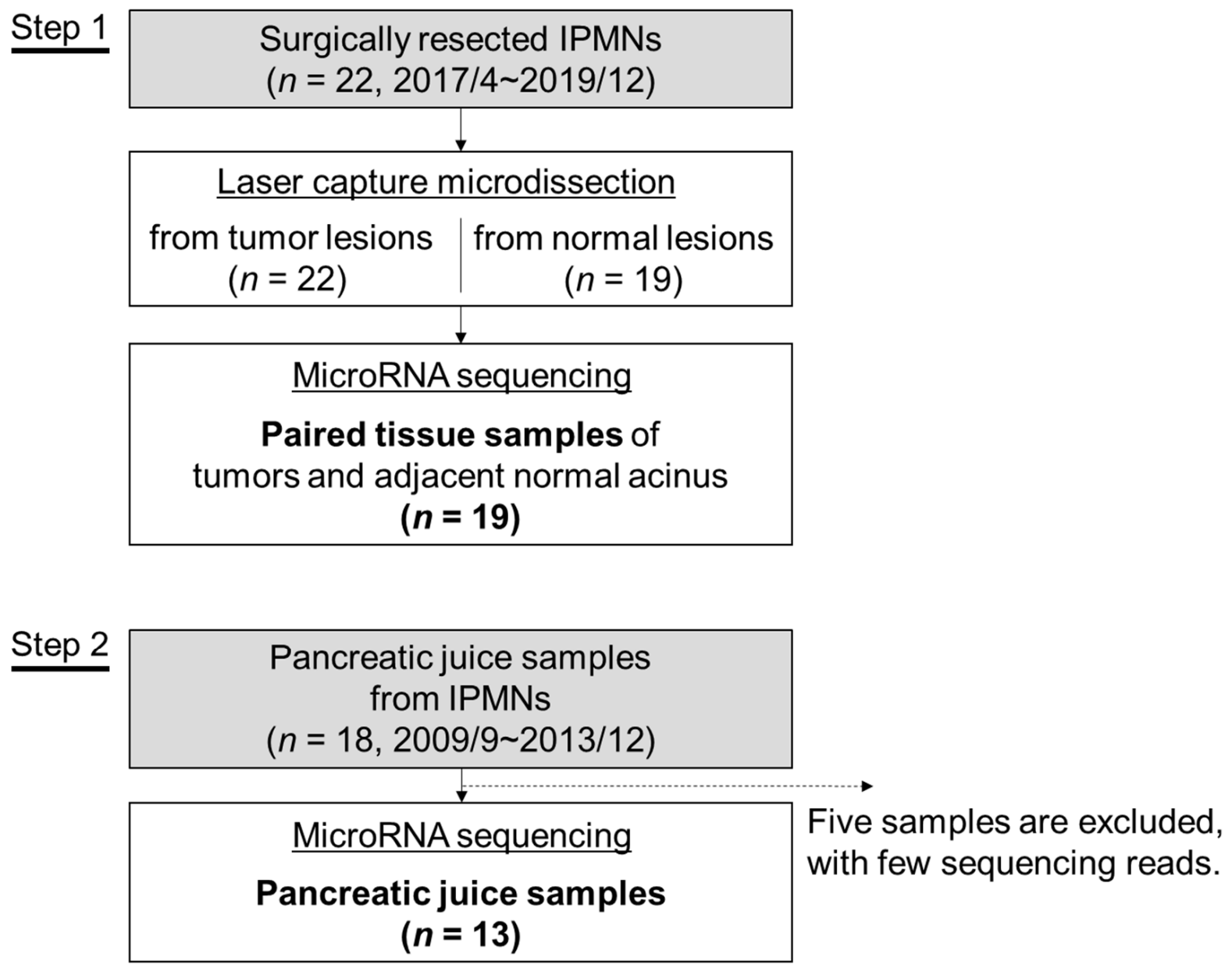

Figure 5. A flowchart of the study. The microRNA expression in intraductal papillary mucinous neoplasm (IPMN) was analyzed in tissue samples (Step 1) and pancreatic juice samples (Step 2). 
Table 2. Summary of clinicopathological features.

\begin{tabular}{lcc}
\hline & Tissue & Pancreatic Juice \\
\hline Age, median (range), years & $(\boldsymbol{n = 1 9 )}$ & $(\boldsymbol{n}=\mathbf{1 3})$ \\
Sex, $n$ (\%) & $74(54-84)$ & $74(61-79)$ \\
Male & $12(63)$ & $5(38)$ \\
Female & $7(37)$ & $8(62)$ \\
Morphological classification, $n(\%)$ & & \\
Branch-duct type & $10(53)$ & $5(38)$ \\
Main-duct type & $4(21)$ & $4(31)$ \\
Mixed type & $5(26)$ & $4(31)$ \\
Grade of IPMN, $n$ (\%) & & $8(62)$ \\
Low-grade dysplasia & $4(21)$ & $2(15)$ \\
High-grade dysplasia & $9(47)$ & $3(23)$ \\
Invasive carcinoma & $6(32)$ & $2 / 1$ \\
UICC stage of invasive carcinoma, $n$ & & \\
IIA/IIB & $1 / 5$ & $9(69)$ \\
Epithelial type of IPMN, $n(\%)$ & & $3(23)$ \\
Gastric & $6(32)$ & $1(8)$ \\
Intestinal & $2(11)$ & 0 \\
Pancreatobiliary & $1(5)$ & 0 \\
Oncocytic & $1(5)$ & \\
Unknown & $9(47)$ & \\
\hline
\end{tabular}

\subsection{RNA Sequencing for miRNA Expression Analysis}

The extracted total RNA or miRNA was enriched for small RNA using a Magnetic Bead Cleanup Module (Thermo Fisher, Waltham, MA, USA), as per the manufacturer's instruction. The quality and quantity of the enriched small RNAs were assessed by the Agilent 2100 Bioanalyzer instrument with the Agilent Small RNA Kit (Agilent Technologies, Santa Clara, CA, USA). Then, the small RNAs were reverse-transcribed using the Invitrogen $^{\mathrm{TM}}$ SuperScript ${ }^{\mathrm{TM}}$ VILO $^{\mathrm{TM}}$ cDNA Synthesis Kit (Thermo Fisher, Waltham, MA, USA). Small RNA libraries for sequencing were generated using the Ion Total RNA-Seq Kit v2 (Thermo Fisher, Waltham, MA, USA) with the Ion Xpress Barcode Adapters Kit (Thermo Fisher, Waltham, MA, USA) for multiplexing samples. Barcoded libraries were amplified using an emulsion polymerase chain reaction on Ion Sphere particles, and sequencing was performed on an Ion Chef System and an Ion Proton Sequencer (Life Technologies, Carlsbad, CA, USA) using an Ion PI Hi-Q Chef Kit (Life Technologies, Carlsbad, CA, USA). Sequenced reads were trimmed, their quality assessed by a program called FastQC, aligned to the reference genome (hg19) and then counted for miRNA using the SmallRNA Analysis program on the Torrent Suite Software (Thermo Fisher, Waltham, MA, USA).

\subsection{Analysis of Count Data for Differentially Expressed miRNAs}

The count data for each miRNA were normalized by calculating the percentage of each miRNA count in the total sample as follows: "Normalized miRNA expression $=($ Read count of each miRNA)/(All read counts in the sample)." The tumor-to-normal expression ratio was calculated as follows: "Normalized miRNA expression of the tumor tissue samples/Normalized miRNA expression of the adjacent normal acinus tissue samples." The count data in pancreatic juice samples were normalized and analyzed without a normal denominator.

A heatmap of miRNA expressions with pathological grades of IPMN was created by showing the miRNA expression levels on a green-red scale in a panel where the green and red scales indicated a low and high expression, respectively. Patients and miRNAs were arranged according to an unsupervised hierarchical clustering, which was performed using a one minus Pearson's correlation as the distance measure and an average linkage as the agglomerative method. 


\subsection{Statistical Analysis}

Comparisons of the miRNA expression within groups and between tumor tissues and their adjacent normal acinus were evaluated using the Kruskal-Wallis test and MannWhitney U test, respectively, and $p<0.05$ was considered significant. All statistical analyses were performed using the statistical functions built into the Excel software (Microsoft Corporation, Redmond, WA, USA), and a volcano plot analysis was made by plotting the significance versus fold-change on the $\mathrm{y}$ - and $x$-axis, respectively.

Supplementary Materials: The following are available online at https:/ /www.mdpi.com/1422-006 7/22/6/3221/s1. Table S1: Summary of aligned reads of RNA sequences for miRNA expressions from tissue and pancreatic juice samples; Table S2: Detailed patients' characteristics; Figure S1: Representative pathological images of LGD, HGD and invasive IPMN (INV).

Author Contributions: Conceptualization, S.T. and N.K.; Methodology, N.E.; Software, S.T. and N.K.; Validation, Y.F., S.K., S.H. and H.H.; Formal Analysis, N.K.; Writing-Original Draft Preparation, S.T. and N.K.; Writing-Review \& Editing, S.M., M.K., H.S., E.T., H.T., N.N., R.K., Y.N., H.K. (Hiromichi Kawaida) and H.K. (Hiroschi Kono); Visualization, S.T. and N.K.; Supervision, T.K., D.I. and N.E.; Project Administration, S.M., T.Y. and T.I.; Funding Acquisition, S.T. and M.F. All authors have read and agreed to the published version of the manuscript.

Funding: This study was supported by grants from the Japan Society for the Promotion of Science (JSPS KAKENHI Grant Numbers: 19K08418, 18K07999 and 15K09044; http:/ /www.jsps.go.jp/jgrantsinaid/).

Institutional Review Board Statement: This study was approved by the Human Ethics Review Committee of Yamanashi University Hospital (Receipt numbers: 1326 and 1847).

Informed Consent Statement: Informed consent was obtained from all subjects involved in the study.

Data Availability Statement: Research data obtained in this study are not shared.

Acknowledgments: We thank Takako Ohmori and Tomoko Nakajima for their valuable technical assistance.

Conflicts of Interest: There are no conflicts of interest to declare.

\section{References}

1. Basturk, O.; Hong, S.M.; Wood, L.D.; Adsay, N.V.; Albores-Saavedra, J.; Biankin, A.V.; Brosens, L.A.; Fukushima, N.; Goggins, M.; Hruban, R.H.; et al. A revised classification system and recommendations from the baltimore consensus meeting for neoplastic precursor lesions in the pancreas. Am. J. Surg. Pathol. 2015, 39, 1730-1741. [CrossRef]

2. Hruban, R.H.; Takaori, K.; Klimstra, D.S.; Adsay, N.V.; Albores-Saavedra, J.; Biankin, A.V.; Biankin, S.A.; Compton, C.; Fukushima, N.; Furukawa, T.; et al. An illustrated consensus on the classification of pancreatic intraepithelial neoplasia and intraductal papillary mucinous neoplasms. Am. J. Surg. Pathol. 2004, 28, 977-987. [CrossRef] [PubMed]

3. Zerboni, G.; Signoretti, M.; Crippa, S.; Falconi, M.; Arcidiacono, P.G.; Capurso, G. Systematic review and meta-analysis: Prevalence of incidentally detected pancreatic cystic lesions in asymptomatic individuals. Pancreatology 2019, 19, 2-9. [CrossRef]

4. Vege, S.S.; Ziring, B.; Jain, R.; Moayyedi, P. American gastroenterological association institute guideline on the diagnosis and management of asymptomatic neoplastic pancreatic cysts. Gastroenterology 2015, 148, 819-822. [CrossRef]

5. Adsay, N.V.; Fukushima, N.; Furukawa, T.; Hruban, R.H.; Klimstra, D.S.; Kloppel, G. Intraductal Neoplasm of the Pancreas; Bosman, F.T., Carneiro, F., Hruban, R.H., Theise, N.D., Eds.; WHO Press: Lyon, France, 2010.

6. Mino-Kenudson, M.; Fernández-del Castillo, C.; Baba, Y.; Valsangkar, N.P.; Liss, A.S.; Hsu, M.; Correa-Gallego, C.; Ingkakul, T.; Johnston, R.P.; Turner, B.G.; et al. Prognosis of invasive intraductal papillary mucinous neoplasm depends on histological and precursor epithelial subtypes. Gut 2011, 60, 1712-1720. [CrossRef]

7. Poultsides, G.A.; Reddy, S.; Cameron, J.L.; Hruban, R.H.; Pawlik, T.M.; Ahuja, N.; Jain, A.; Edil, B.H.; Iacobuzio-Donahue, C.A.; Schulick, R.D.; et al. Histopathologic basis for the favorable survival after resection of intraductal papillary mucinous neoplasmassociated invasive adenocarcinoma of the pancreas. Ann. Surg. 2010, 251, 470-476. [CrossRef]

8. Rezaee, N.; Barbon, C.; Zaki, A.; He, J.; Salman, B.; Hruban, R.H.; Cameron, J.L.; Herman, J.M.; Ahuja, N.; Lennon, A.M.; et al. Intraductal papillary mucinous neoplasm (IPMN) with high-grade dysplasia is a risk factor for the subsequent development of pancreatic ductal adenocarcinoma. НРВ 2016, 18, 236-246. [CrossRef]

9. Tanaka, M.; Chari, S.; Adsay, V.; Fernandez-del Castillo, C.; Falconi, M.; Shimizu, M.; Yamaguchi, K.; Yamao, K.; Matsuno, S.; International Association of Pancreatology. International consensus guidelines for management of intraductal papillary mucinous neoplasms and mucinous cystic neoplasms of the pancreas. Pancreatology 2006, 6, 17-32. [CrossRef] 
10. Tanaka, M.; Fernandez-del Castillo, C.; Adsay, V.; Chari, S.; Falconi, M.; Jang, J.Y.; Kimura, W.; Levy, P.; Pitman, M.B.; Schmidt, C.M.; et al. International consensus guidelines 2012 for the management of IPMN and MCN of the pancreas. Pancreatology 2012, 12, 183-197. [CrossRef]

11. Tanaka, M.; Fernández-del Castillo, C.; Kamisawa, T.; Jang, J.Y.; Levy, P.; Ohtsuka, T.; Salvia, R.; Shimizu, Y.; Tada, M.; Wolfgang, C.L. Revisions of international consensus Fukuoka guidelines for the management of IPMN of the pancreas. Pancreatology 2017, 17, 738-753. [CrossRef]

12. Sharib, J.M.; Fonseca, A.L.; Swords, D.S.; Jaradeh, K.; Bracci, P.M.; Firpo, M.A.; Hatcher, S.; Scaife, C.L.; Wang, H.; Kim, G.E.; et al. Surgical overtreatment of pancreatic intraductal papillary mucinous neoplasms: Do the 2017 International Consensus Guidelines improve clinical decision making? Surgery 2018, 164, 1178-1184. [CrossRef]

13. Aso, T.; Ohtsuka, T.; Matsunaga, T.; Kimura, H.; Watanabe, Y.; Tamura, K.; Ideno, N.; Osoegawa, T.; Takahata, S.; Shindo, K.; et al. "High-risk stigmata" of the 2012 international consensus guidelines correlate with the malignant grade of branch duct intraductal papillary mucinous neoplasms of the pancreas. Pancreas 2014, 43, 1239-1243. [CrossRef]

14. Goh, B.K.; Lin, Z.; Tan, D.M.; Thng, C.H.; Khor, C.J.; Lim, T.K.; Ooi, L.L.; Chung, A.Y. Evaluation of the Fukuoka Consensus Guidelines for intraductal papillary mucinous neoplasms of the pancreas: Results from a systematic review of 1382 surgically resected patients. Surgery 2015, 158, 1192-1202. [CrossRef] [PubMed]

15. Yang, D.J.; Xiong, J.J.; Liu, X.T.; Li, J.; Siriwardena, K.M.D.L.; Hu, W.M. Total pancreatectomy compared with pancreaticoduodenectomy: A systematic review and meta-analysis. Cancer Manag. Res. 2019, 11, 3899-3908. [CrossRef] [PubMed]

16. Barbier, L.; Jamal, W.; Dokmak, S.; Aussilhou, B.; Corcos, O.; Ruszniewski, P.; Belghiti, J.; Sauvanet, A. Impact of total pancreatectomy: Short- and long-term assessment. HPB 2013, 15, 882-892. [CrossRef] [PubMed]

17. Beger, H.G.; Mayer, B.; Poch, B. Parenchyma-sparing, local pancreatic head resection for premalignant and low-malignant neoplasms-A systematic review and meta-analysis. Am. J. Surg. 2018, 216, 1182-1191. [CrossRef]

18. Takano, S.; Fukasawa, M.; Kadokura, M.; Shindo, H.; Takahashi, E.; Hirose, S.; Maekawa, S.; Mochizuki, K.; Kawaida, H.; Itakura, J.; et al. Next-generation sequencing revealed TP53 mutations to be malignant marker for intraductal papillary mucinous neoplasms that could be detected using pancreatic juice. Pancreas 2017. [CrossRef]

19. Kanda, M.; Knight, S.; Topazian, M.; Syngal, S.; Farrell, J.; Lee, J.; Kamel, I.; Lennon, A.M.; Borges, M.; Young, A.; et al. Mutant GNAS detected in duodenal collections of secretin-stimulated pancreatic juice indicates the presence or emergence of pancreatic cysts. Gut 2012, 62, 1024-1033. [CrossRef]

20. Wu, J.; Matthaei, H.; Maitra, A.; Dal Molin, M.; Wood, L.D.; Eshleman, J.R.; Goggins, M.; Canto, M.I.; Schulick, R.D.; Edil, B.H.; et al. Recurrent GNAS mutations define an unexpected pathway for pancreatic cyst development. Sci. Transl. Med. 2011, 3, 92ra66. [CrossRef]

21. Mitchell, P.S.; Parkin, R.K.; Kroh, E.M.; Fritz, B.R.; Wyman, S.K.; Pogosova-Agadjanyan, E.L.; Peterson, A.; Noteboom, J.; O'Briant, K.C.; Allen, A.; et al. Circulating microRNAs as stable blood-based markers for cancer detection. Proc. Natl. Acad. Sci. USA 2008, 105, 10513-10518. [CrossRef]

22. Chen, X.; Ba, Y.; Ma, L.; Cai, X.; Yin, Y.; Wang, K.; Guo, J.; Zhang, Y.; Chen, J.; Guo, X.; et al. Characterization of microRNAs in serum: A novel class of biomarkers for diagnosis of cancer and other diseases. Cell Res. 2008, 18, 997-1006. [CrossRef]

23. Bartel, D.P. MicroRNAs: Target recognition and regulatory functions. Cell 2009, 136, 215-233. [CrossRef]

24. Jiang, C.; Chen, X.; Alattar, M.; Wei, J.; Liu, H. MicroRNAs in tumorigenesis, metastasis, diagnosis and prognosis of gastric cancer. Cancer Gene Ther. 2015, 22, 291-301. [CrossRef] [PubMed]

25. Frampton, A.E.; Castellano, L.; Colombo, T.; Giovannetti, E.; Krell, J.; Jacob, J.; Pellegrino, L.; Roca-Alonso, L.; Funel, N.; Gall, T.M.H.; et al. MicroRNAs cooperatively inhibit a network of tumor suppressor genes to promote pancreatic tumor growth and progression. Gastroenterology 2014, 146. [CrossRef]

26. Hamada, S.; Satoh, K.; Miura, S.; Hirota, M.; Kanno, A.; Masamune, A.; Kikuta, K.; Kume, K.; Unno, J.; Egawa, S.; et al. miR-197 induces epithelial-mesenchymal transition in pancreatic cancer cells by targeting p120 catenin. J. Cell. Physiol. 2013, 228, 1255-1263. [CrossRef] [PubMed]

27. Nishiwada, S.; Sho, M.; Banwait, J.K.; Yamamura, K.; Akahori, T.; Nakamura, K.; Baba, H.; Goel, A. A MicroRNA signature identifies pancreatic ductal adenocarcinoma patients at risk for lymph node metastases. Gastroenterology 2020, 159, 562-574. [CrossRef] [PubMed]

28. Abue, M.; Yokoyama, M.; Shibuya, R.; Tamai, K.; Yamaguchi, K.; Sato, I.; Tanaka, N.; Hamada, S.; Shimosegawa, T.; Sugamura, K.; et al. Circulating miR-483-3p and miR-21 is highly expressed in plasma of pancreatic cancer. Int. J. Oncol. 2015, 46, 539-547. [CrossRef] [PubMed]

29. Nakamura, S.; Sadakari, Y.; Ohtsuka, T.; Okayama, T.; Nakashima, Y.; Gotoh, Y.; Saeki, K.; Mori, Y.; Nakata, K.; Miyasaka, Y.; et al. Pancreatic juice exosomal microRNAs as biomarkers for detection of pancreatic ductal adenocarcinoma. Ann. Surg. Oncol. 2019, 26, 2104-2111. [CrossRef] [PubMed]

30. Zhao, F.; Wei, C.; Cui, M.Y.; Xia, Q.Q.; Wang, S.B.; Zhang, Y. Prognostic value of microRNAs in pancreatic cancer: A meta-analysis. Aging 2020, 12, 9380-9404. [CrossRef]

31. Habbe, N.; Koorstra, J.B.M.; Mendell, J.T.; Offerhaus, G.J.; Ji, K.R.; Feldmann, G.; Mullendore, M.E.; Goggins, M.G.; Hong, S.M.; Maitra, A. MicroRNA miR-155 is a biomarker of early pancreatic neoplasia. Cancer Biol. Ther. 2009, 8, 340-346. [CrossRef] 
32. Caponi, S.; Funel, N.; Frampton, A.E.; Mosca, F.; Santarpia, L.; Van der Velde, A.G.; Jiao, L.R.; De Lio, N.; Falcone, A.; Kazemier, G.; et al. The good, the bad and the ugly: A tale of miR-101, miR-21 and miR-155 in pancreatic intraductal papillary mucinous neoplasms. Ann. Oncol. 2013, 24, 734-741. [CrossRef] [PubMed]

33. Lubezky, N.; Loewenstein, S.; Ben-Haim, M.; Brazowski, E.; Marmor, S.; Pasmanik-Chor, M.; Oron-Karni, V.; Rechavi, G.; Klausner, J.M.; Lahat, G. MicroRNA expression signatures in intraductal papillary mucinous neoplasm of the pancreas. Surgery 2013, 153, 663-672. [CrossRef] [PubMed]

34. Goto, T.; Fujiya, M.; Konishi, H.; Sasajima, J.; Fujibayashi, S.; Hayashi, A.; Utsumi, T.; Sato, H.; Iwama, T.; Ijiri, M.; et al. An elevated expression of serum exosomal microRNA-191, -21, -451a of pancreatic neoplasm is considered to be efficient diagnostic marker. BMC Cancer 2018, 18. [CrossRef]

35. Permuth-Wey, J.; Chen, Y.A.; Fisher, K.; McCarthy, S.; Qu, X.; Lloyd, M.C.; Kasprzak, A.; Fournier, M.; Williams, V.L.; Ghia, K.M.; et al. A genome-wide investigation of microRNA expression identifies biologically-meaningful microRNAs that distinguish between high-risk and low-risk intraductal papillary mucinous neoplasms of the pancreas. PLoS ONE 2015, 10, e0116869. [CrossRef]

36. Wang, J.; Paris, P.L.; Chen, J.; Ngo, V.; Yao, H.; Frazier, M.L.; Killary, A.M.; Liu, C.G.; Liang, H.; Mathy, C.; et al. Next generation sequencing of pancreatic cyst fluid microRNAs from low grade-benign and high grade-invasive lesions. Cancer Lett. 2015, 356, 404-409. [CrossRef] [PubMed]

37. Bloomston, M.; Frankel, W.L.; Petrocca, F.; Volinia, S.; Alder, H.; Hagan, J.P.; Liu, C.G.; Bhatt, D.; Taccioli, C.; Croce, C.M. MicroRNA expression patterns to differentiate pancreatic adenocarcinoma from normal pancreas and chronic pancreatitis. J. Am. Med. Assoc. 2007, 297, 1901-1908. [CrossRef]

38. Chen, W.; Tang, Z.; Sun, Y.; Zhang, Y.; Wang, X.; Shen, Z.; Liu, F.; Qin, X. miRNA expression profile in primary gastric cancers and paired lymph node metastases indicates that miR-10a plays a role in metastasis from primary gastric cancer to lymph nodes. Exp. Ther. Med. 2012, 3, 351-356. [CrossRef]

39. Grolmusz, V.K.; Kövesdi, A.; Borka, K.; Igaz, P.; Patócs, A. Prognostic relevance of proliferation-related miRNAs in pancreatic neuroendocrine neoplasms. Eur. J. Endocrinol. 2018, 179, 219-228. [CrossRef]

40. Cohen, S.J.; Papoulas, M.; Graubardt, N.; Ovdat, E.; Loewenstein, S.; Kania-Almog, J.; Pasmanik-Chor, M.; Brazowski, E.; Cagnano, E.; Nachmany, I.; et al. Micro-RNA Expression patterns predict metastatic spread in solid pseudopapillary neoplasms of the pancreas. Front. Oncol. 2020, 10. [CrossRef]

41. Yu, T.; Liu, L.; Li, J.; Yan, M.; Lin, H.; Liu, Y.; Chu, D.; Tu, H.; Gu, A.; Yao, M. MiRNA-10a is upregulated in NSCLC and may promote cancer by targeting PTEN. Oncotarget 2015, 6, 30239-30250. [CrossRef]

42. Zeng, T.; Li, G. MicroRNA-10a enhances the metastatic potential of cervical cancer cells by targeting phosphatase and tensin homologue. Mol. Med. Rep. 2014, 10, 1377-1382. [CrossRef]

43. Ohuchida, K.; Mizumoto, K.; Lin, C.; Yamaguchi, H.; Ohtsuka, T.; Sato, N.; Toma, H.; Nakamura, M.; Nagai, E.; Hashizume, M.; et al. MicroRNA-10a is overexpressed in human pancreatic cancer and involved in its invasiveness partially via suppression of the HOXA1 gene. Ann. Surg. Oncol. 2012, 19, 2394-2402. [CrossRef]

44. Yang, W.; Yang, Y.; Xia, L.; Yang, Y.; Wang, F.; Song, M.; Chen, X.; Liu, J.; Song, Y.; Zhao, Y.; et al. MiR-221 promotes capan2 pancreatic ductal adenocarcinoma cells proliferation by targeting PTEN-Akt. Cell. Physiol. Biochem. 2016, 38, 2366-2374. [CrossRef]

45. Sarkar, S.; Dubaybo, H.; Ali, S.; Goncalves, P.; Kollepara, S.L.; Sethi, S.; Philip, P.A.; Li, Y. Down-regulation of miR-221 inhibits proliferation of pancreatic cancer cells through up-regulation of PTEN, p27(kip1), p57(kip2), and PUMA. Am. J. Cancer Res. 2013, 3, 465-477.

46. Wu, X.; Huang, J.; Yang, Z.; Zhu, Y.; Zhang, Y.; Wang, J.; Yao, W. MicroRNA-221-3p is related to survival and promotes tumour progression in pancreatic cancer: A comprehensive study on functions and clinicopathological value. Cancer Cell Int. 2020, 20. [CrossRef]

47. Zhi, Y.; Xie, X.; Wang, R.; Wang, B.; Gu, W.; Ling, Y.; Dong, W.; Zhi, F.; Liu, Y. Serum level of miR-10-5p as a prognostic biomarker for acute myeloid leukemia. Int. J. Hematol. 2015, 102, 296-303. [CrossRef] [PubMed]

48. Safari, A.; Seifoleslami, M.; Yahaghi, E.; Sedaghati, F.; Khameneie, M.K. RETRACTED ARTICLE: Upregulation of miR-20a and miR-10a expression levels act as potential biomarkers of aggressive progression and poor prognosis in cervical cancer. Tumor Biol. 2016, 37, 16387. [CrossRef] [PubMed]

49. Duell, E.J.; Lujan-Barroso, L.; Sala, N.; McElyea, S.D.; Overvad, K.; Tjonneland, A.; Olsen, A.; Weiderpass, E.; Busund, L.T.; Moi, L.; et al. Plasma microRNAs as biomarkers of pancreatic cancer risk in a prospective cohort study. Int. J. Cancer 2017, 141, 905-915. [CrossRef] [PubMed]

50. Ma, M.Z.; Kong, X.; Weng, M.Z.; Cheng, K.; Gong, W.; Quan, Z.W.; Peng, C.H. Candidate microRNA biomarkers of pancreatic ductal adenocarcinoma: Meta-analysis, experimental validation and clinical significance. J. Exp. Clin. Cancer Res. $2013,32$. [CrossRef] 